\title{
About the use of micro-indentation to determine the constitutive material's mechanical behaviour of cellular structures
}

\author{
V. Marcadon ${ }^{1, a}$, B. Passilly ${ }^{1}$, C. Davoine ${ }^{1}$, A. Götzfried ${ }^{1}$, and F.-H. Leroy ${ }^{1}$ \\ ONERA, 29 Av. de la Division Leclerc, BP 72, 92322 Châtillon Cedex, France
}

\begin{abstract}
Architectured cellular materials seem to be very promising materials since they can combine interesting specific mechanical, acoustical and thermal properties. Nevertheless the process routes used to elaborate such materials, especially the thermal treatments and the added compounds, may alter their constitutive materials for which the mechanical behaviour becomes unknown. In order to determine locally the mechanical behaviour of the constitutive materials of cellular structures we propose the use of microindentation. The model structures considered here are brazed tube stackings. Macroscopic tensile tests and micro-indentation measurements have been performed on isolated tubes having been submitted to different thermal treatment durations. The mechanical properties estimated thanks to both methods show a good agreement. These results also put in evidence the strong influence of the thermal treatment on the behaviour of the constitutive material of the tubes. Micro-indentation measurements performed in the braze joints gave access to their mechanical properties which are strongly heterogeneous. A comparison between the experimental mechanical response of the tube stackings under compression and the one predicted by the finite-element method is proposed too.
\end{abstract}

\section{Introduction}

For many years cellular architectured structures, like honeycombs, metal foams or hollow-sphere stackings, have been abundantly studied because they could combine multi-functionalities by optimizing the cell size, shape or arrangement. Especially, they could represent an alternative solution to bulk materials for numerous structural applications like impact resistance for example, and to develop lightweight aeronautical frames since higher specific properties than bulk are expected [1]. In the literature numerous studies deal with the role of the architecture of cellular structures (cell geometry and arrangement) on their effective mechanical behaviour, one can cite for example [2-5] as concerns hollow-sphere stackings. Some original approaches, coupling X-Ray tomography characterization and the finite-element method for example [6], have been proposed to capture as accurately as possible the complex architecture of such materials. However, the issue of the mechanical properties of the constitutive material, or often the materials, and its influence on the cellular material's effective behaviour, is rarely tackled with. For example in the case of metallic hollow-sphere stackings processed by sintering metallic powders many residual porosities are observed in the walls of the spheres [5,6]. Caty et al. [6] have raised the issue of the influence of porosities on the mechanical behaviour of the constitutive material of the stackings to explain the required adjustment of the mechanical properties of the constitutive material to achieve a good agreement between the experiment and the modelling of such materials. Such an effect of the porosities has been asserted by Marcadon and Feyel [7] using a micromechanical approach. In the case of brazed materials, similar effects of the process route are expected because of the brazing material's elements which diffuse in the walls of the cells during the brazing treatment.

\footnotetext{
a e-mail: Vincent.Marcadon@onera.fr
} 
Because of both the elaboration process and thermal treatments applied to elaborate the constitutive cells and to assemble them together, the constitutive material's behaviour is often unknown. To propose a reliable method to characterize the in-situ mechanical properties of the constitutive materials of cellular structures is thus critical to improve the predictive modelling of their overall mechanical properties. To address this issue a coupled approach involving both micro-indentation tests and classical macroscopic ones is proposed to evaluate the relevance of using micro-indentation to determine the local mechanical properties of the different constitutive phases of the walls of cellular structures. First the model materials studied, which consist in stackings of tubes brazed together, and their process route are described. Then the second part is dedicated to the comparison between the mechanical properties of the constitutive material of the tubes estimated from both the macroscopic tensile tests and the micro-indentation measurements. The effect of the thermal treatment on these properties is studied. Heterogeneity of the braze joints and diffusion of the brazing compounds in the tubes, and their influence on the local mechanical properties, are discussed too. The last part proposes a preliminary comparison between the overall mechanical behaviour predicted by the finite-element method and the one experimentally obtained by performing compression tests on small sandwich structures.

\section{A model cellular structure}

Small sandwich structures have been processed using a brazing thermal treatment. They consisted of two Inconel ${ }^{\mathbb{R}} 600$ sheets of $200 \mu \mathrm{m}$ thickness and a tube stacking core. The tubes were in Inconel ${ }^{\circledR}$ 600 too and their outer radius and their thickness equalled $2 \mathrm{~mm}$ and $200 \mu \mathrm{m}$, respectively. They were linked together and to the sheets using a Nickel-Phosphorus braze. Scanning Electron Microscopy (SEM) revealed that the joint width was around $700 \mu \mathrm{m}$. Two types of stackings following either a square or an hexagonal pattern in its transversal plan (figs. 1(a) and 1(b)) have been produced. Sandwiches were four-tube large and five-tube high. The length of the tubes equalled $9.6 \mathrm{~mm}$.

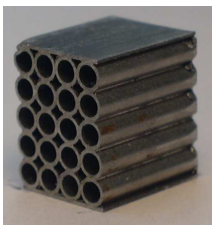

(a)

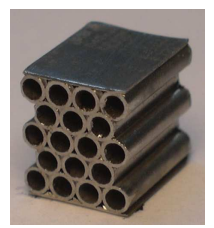

(b)

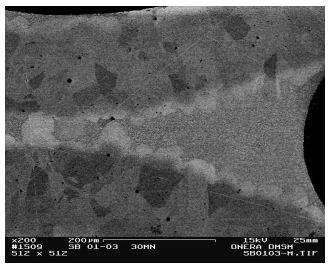

(c)

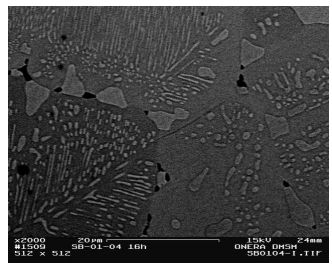

(d)

Fig. 1. Sandwich structure with a) a square stacking core b) an hexagonal stacking core c) SEM of a braze joint (medium grey) linking two neighbour tubes d) zoom on the eutectic structure in the joint.

Nickel-Phosphorus (Ni-P) is widely used as an electroless coating in different industries because of both its corrosion and abrasion resistance properties, and its uniform coating thickness. Here Ni-P is used as brazing material. A $15 \mu \mathrm{m}$ thick Ni-P layer was deposited on the surface of each tube by electroless plating. Before immersion in the electroless Ni-P deposition bath, the tubes were cleaned in an alkali solution to remove dirts or greases on the surface, and etched in nitric acid to remove the native oxide layer. A commercial electroless nickel bath which contains nickel sulfate as a $\mathrm{Ni}$ ion source and sodium hypophosphite as a reducing agent was used to obtain the coating. This bath provides Ni-P deposits with a medium phosphorus content, 6-8 \% in weight (wt.\%). The deposit duration was $90 \mathrm{~min}$. Since the ends of the tubes were not hermetically closed, the coating occurred on the inner surface of the tubes too. Tubes were stacked in a graphite die lined with Inconel ${ }^{\circledR} 600$ sheets. A close-packed arrangement of the tubes is naturally created and provides the "hexagonal stacking". The "square stacking" is obtained by sloping the graphite die in order to use its edges to help the positioning of the tubes. The bonding is achieved by applying the brazing treatment in a vacuum furnace: first an increase of the temperature up to $1050^{\circ} \mathrm{C}$ at a rate of $10^{\circ} \mathrm{C} / \mathrm{min}$, followed by 
a dwell at $1050^{\circ} \mathrm{C}$, then followed by the natural cooling of the furnace. According to the Ni-P phase diagram, a Ni-8 wt.\% P alloy is liquid for a temperature above $1000^{\circ} \mathrm{C}$. Thanks to capillarity the liquid concentrates along the contact line between two neighbour tubes, hence metallurgical joints are created between the tubes during the cooling (fig. 1(c)). Two durations for the thermal treatment have been applied in order to study both the diffusion of phosphorus in Inconel ${ }^{\circledR} 600$ and the homogenization of the microstructure in the braze joint: a short thermal treatment at $1050^{\circ} \mathrm{C}$ during $30 \mathrm{~min}$ and a long one at $1050^{\circ} \mathrm{C}$ during $16 \mathrm{~h}$. Energy Dispersive Spectroscopy analyses showed that the Ni-P eutectic structure consists of two distinct phases, a Ni-rich one and an intermetallic compound $\mathrm{Ni}_{3} \mathrm{P}$ (fig. 1(d)).

\section{Mechanical behaviour of the constitutive material}

\subsection{Influence of the brazing thermal treatment}

On the purpose to determine the mechanical behaviour of the Inconel ${ }^{\circledR} 600$ that constituted the walls of the tubes, but also to study the influence of the brazing thermal treatment on it, uniaxial tensile tests have been conducted on isolated tubes. The reference is the non-treated tube, labelled NTT for "No Thermal Treatment". The tubes having been submitted to the brazing thermal treatments are labelled STT and LTT (for "Short Thermal Treatment" and "Long Thermal Treatment"), for dwell durations of $30 \mathrm{~min}$ and $16 \mathrm{~h}$, respectively. For the three different thermal treatments, NTT, STT and LTT, three tubes have been tested. Experiments have been performed at room temperature using a classical traction-compression machine. The length of the tubes equalled $50 \mathrm{~mm}$ and both ends of the tubes were handled in holding jaws. Small solid tubes of $10 \mathrm{~mm}$ length were introduced in the ends of the tubes to allow their holding and to avoid their collapse during the tests, which would have altered the measurement of the elongation. Experiments were governed in displacement at a rate of $0.5 \mathrm{~mm} / \mathrm{min}$. The longitudinal elongation of the tubes was measured thanks to an extensometer placed at the centre of the sample. Stress $v s$. strain curves are plotted in figures 2(a) and 2(b). They show the very good reproducibility of the tests. But the main conclusion of these tests is the fact that the thermal treatment drastically decreases the strength of the tubes (fig. 3(a)). Hence the mechanical behaviour of the constitutive material of the tubes is strongly altered by the thermal treatment. Such an effect of the thermal treatment asserts how critical is a method to determine accurately the in-situ mechanical properties of the constitutive materials of cellular structures.

Mechanical properties of the Inconel ${ }^{\circledR} 600$ have been identified for the three different thermal treatments by fitting the stress $v s$. strain curves with the mechanical response of an Homogeneous Equivalent Medium (HEM). For each treatment, the fitted curves have been optimized by taking into account the three tests. The constitutive material was assumed to be elasto-plastic isotropic. The elasticity was supposed to be governed according to the Hook's law and a von Mises criterion was chosen for the yield surface. First the hardening was assumed to be linear but such a bi-linear model is not accurate enough to correctly capture the non-linearities observed in the plastic domain (figs. 2(a) and 2(b)). Consequently, a non-linear exponential term has been added to the hardening: $\sigma_{e q}=\sigma_{y m}+H_{m} p+Q_{m}\left(1-e^{-b_{m} p}\right)$, where $\sigma_{e q}$ is the equivalent von Mises stress and $p$ is the cumulated plastic strain. $\sigma_{y m}$ and $H_{m}$ denote the yield stress and the hardening modulus of the constitutive material, respectively, and $Q_{m}$ and $b_{m}$ are the coefficients of the non-linear hardening. The Young's modulus of the constitutive material is noted $E_{m}$. Both elastic and plastic moduli determined for the different treatments are provided in table 1 . The hypothesis that the tangential stress in the tubes is negligible (i.e., the tensile response of an hollow tubes is the same as the one of a solid HEM) has been verified by modelling through the finite-element method (FEM) the traction of the tubes with the identified laws. The resulting curves were superimposed with the ones obtained on the HEM.

It is worth noting that the plastic behaviour of the Inconel ${ }^{\circledR} 600$ strongly varies between before and after the thermal treatment. Whereas before the thermal treatment (NTT) the plastic behaviour is rather close to a perfect plastic one (i.e., a high yield stress and a low hardening modulus), after the thermal treatments (STT and LTT) the material shows a strong hardening (fig. 3(a) and tab. 1). Such an annealing effect of the treatment results from the relaxation of the internal stresses in the tubes processed by wire drawing. Thus the original tubes are saturated in dislocations, hence the high yield 


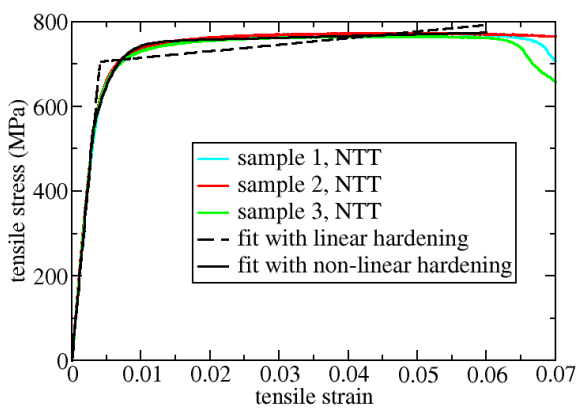

(a)

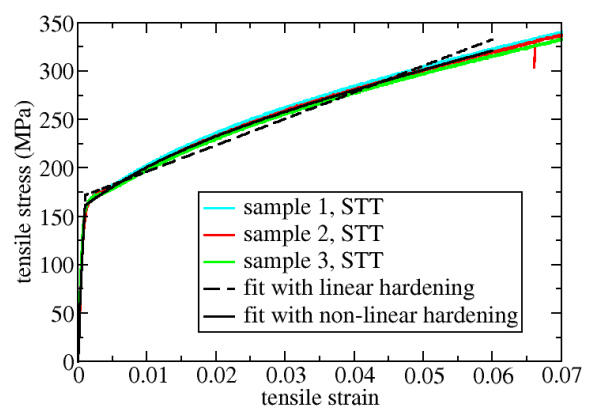

(b)

Fig. 2. Stress $v s$. strain curves resulting from the tensile tests performed on the tubes a) non-treated b) submitted to the treatment STT. The fitted behaviours, with both the linear and the non-linear hardening, are plotted in the figures. Similar curves have been obtained for the thermal treatment LTT.

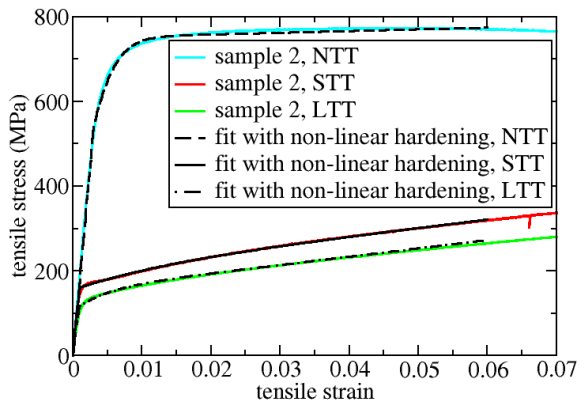

(a)

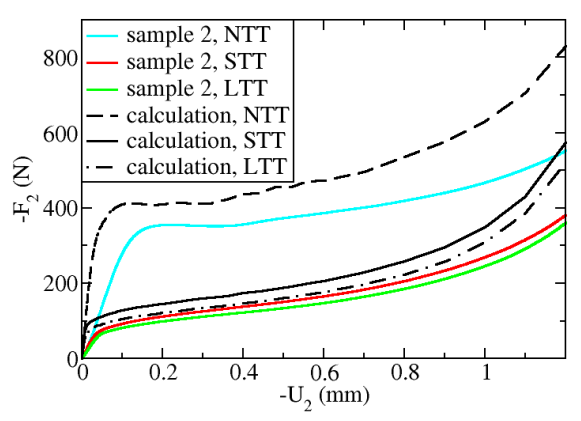

(b)

Fig. 3. Influence of the treatment on a) the longitudinal strength of the tubes in tension b) the transversal strength of the tubes in compression. $F_{2}$ and $U_{2}$ are the force and the displacement transversally to the axis of the tubes.

stress and the impossibility to harden more the material. The high temperature decreases the density of dislocations in the material and allows a possible work-hardening again.

To address the assumption of the constitutive material isotropy, some transversal compression experiments have been carried out on small tubes of $10 \mathrm{~mm}$ length. For each thermal treatment three samples have been tested. In parallel, these compression tests have been simulated using the FEM and the isotropic laws identified from the tensile tests. Obviously, the laws with the non-linear hardening have been considered since they are the most relevant. Compression experiments have shown a very good reproducibility too and have asserted the significant effect of the thermal treatment on the strength of the tubes. They have been compared with the ones predicted by the FEM calculations (see fig. 3(b)). There is a quite good agreement between the experimental and the predicted curves for the treatments STT and LTT. On the contrary, the gap between the calculation and the experiment seems to be larger in the case of the non-treated tubes. Such results could be partially explained by the fact that, whereas the process route of the tubes involves an anisotropy of the Inconel ${ }^{\mathbb{R}} 600$ (the longitudinal stiffness would be significantly higher than the transversal one), the thermal treatment restores the isotropy of the material. Both Electron Backscatter Diffraction and texture analyses are in progress to address in detail the issue of the microstructure of the constitutive material of the tubes, and its influence on the 
mechanical properties. The decrease of the yield stress by increasing the dwell duration might result from an increase of the grain size. Moreover, for the moment there is no explanation for the drastic drop of the Young's modulus of the constitutive material highlighted for the treatment LTT.

\subsection{Micro-indentation vs. tensile tests}

Micro-indentation is expected to be able to provide information on the local mechanical properties of the material which constitutes the stackings by stressing a small volume of the material only. Microhardness measurements have been performed first in the thickness of the isolated tubes using a Vickers indentor. The three different thermal treatments have been considered. Each time, around 20 measurements have been done in the cross section, and results were slightly scattered.

Table 1. Influence of the thermal treatment on the mechanical properties of the Inconel ${ }^{\circledR} 600$ identified from both the tensile and the micro-indentation experiments.

\begin{tabular}{llllll}
\hline & $\begin{array}{l}\text { Tensile tests } \\
\text { linear hardening }\end{array}$ & non-linear hardening & $\sigma_{0.07}$ & $\begin{array}{l}\text { Micro-indentation } \\
\text { Vickers hardness }\end{array}$ & estimated properties \\
\hline NTT & $E_{m}=174.3 \mathrm{GPa}$ & $E_{m}=179.9 \mathrm{GPa}$ & $709.7 \mathrm{MPa}$ & $H_{v m}=2.3 \mathrm{GPa}$ & $E_{m}=212.0 \mathrm{GPa}$ \\
& $\sigma_{y m}=706.1 \mathrm{MPa}$ & $\sigma_{y m}=543.6 \mathrm{MPa}$ & & & $\sigma_{0.08}=824.4 \mathrm{MPa}$ \\
& $H_{m}=1566.8 \mathrm{MPa}$ & $H_{m}=406.2 \mathrm{MPa}$ & & & $\sigma_{0.07}=793.6 \mathrm{MPa}$ \\
& & $Q_{m}=208.5 \mathrm{MPa}$ & & & \\
& & $b_{m}=492.0$ & & & $E_{m}=173.0 \mathrm{GPa}$ \\
STT & $E_{m}=169.0 \mathrm{GPa}$ & $E_{m}=171.9 \mathrm{GPa}$ & $337.0 \mathrm{MPa}$ & $H_{v m}=1.5 \mathrm{GPa}$ & $\sigma_{0.08}=537.6 \mathrm{MPa}$ \\
& $\sigma_{y m}=172.0 \mathrm{MPa}$ & $\sigma_{y m}=160.8 \mathrm{MPa}$ & & & $\sigma_{0.07}=413.8 \mathrm{MPa}$ \\
& $H_{m}=2772.4 \mathrm{MPa}$ & $H_{m}=1974.0 \mathrm{MPa}$ & & & \\
& & $Q_{m}=46.5 \mathrm{MPa}$ & & & $E_{m}=115.0 \mathrm{GPa}$ \\
& & $b_{m}=76.2$ & & $\sigma_{0.08}=501.8 \mathrm{MPa}$ \\
LTT & $E_{m}=121.9 \mathrm{GPa}$ & $E_{m}=130.2 \mathrm{GPa}$ & $286.0 \mathrm{MPa}$ & $H_{v m}=1.4 \mathrm{GPa}$ & \\
& $\sigma_{y m}=139.7 \mathrm{MPa}$ & $\sigma_{y m}=116.3 \mathrm{MPa}$ & & & $\sigma_{0.07}=375.7 \mathrm{MPa}$ \\
& $H_{m}=2463.5 \mathrm{MPa}$ & $H_{m}=1994.6 \mathrm{MPa}$ & & & \\
& & $Q_{m}=41.3 \mathrm{MPa}$ & & & \\
\hline
\end{tabular}

Obtained hardness values $H_{v m}$ assert the drastic drop of the material's behaviour induced by the thermal treatment (see tab. 1 and fig. 4(a)). Whereas the hardness equals $2.3 \mathrm{GPa}$ for the treatment NTT, it equals $1.5 \mathrm{GPa}$ and $1.4 \mathrm{GPa}$ for treatments STT and LTT, respectively. First estimates of the constitutive material's Young modulus have been made from micro-indentation curves using the method proposed by Oliver and Pharr [8] and the correction proposed by Leroy (and detailed in [9]) to better capture the piling up phenomenon classically observed in materials having a behaviour close to a perfectly plastic one. The moduli are provided in table 1 . It is worth noting that there is a very good agreement between the Young's moduli estimated from both the tensile tests and the micro-indentation ones for the thermal treatments STT and LTT. On the contrary, the Young's modulus estimated from the micro-indentation for the non-treated tube overestimates the one coming from the tensile tests. In the case of a material possessing very low hardening capabilities, the piling up observed around the track results in an underestimation of the contact area between the material and the indentor, hence an overestimation of the Young's modulus. Despite of the correction proposed here, the estimation of the Young's modulus remains rather tricky for the treatment NTT.

In the case of a Vickers indentor, the stresses for a cumulated plastic strain of $8 \%$ and $7 \%$ have been determined from the equations of Tabor [10] and Johnson [11], respectively: $\sigma_{0.08} \approx H_{v m} /(3 \times$ $0.93)$ and $\sigma_{0.07} \approx\left(3 H_{v m} /(2 \times 0.93)\right)\left(1+\ln \left(E_{m} \tan (\pi / 2-70.32 \pi / 180) /\left(3 \sigma_{y m}\right)\right)\right)^{-1}$. Values are given in table 1. $\sigma_{0.07}$ has been estimated from the tensile tests too (tab. 1). For the Johnson's formula, the 
ratio $E_{m} / \sigma_{y m}$ has been computed from the values of $E_{m}$ and $\sigma_{y m}$ identified from the tensile tests. The Tabor's equation, by providing an estimate rather for $\sigma_{0.08}$, strongly overestimates the values of $\sigma_{0.07}$, especially for treatments STT and LTT since it does not suite well in the case of hardening. On the contrary, even if the Johnson's equation overestimates the values of $\sigma_{0.07}$ too, estimated values are rather close to the experimental ones for the three thermal treatments. Such results are encouraging because they highlight the relevance of the micro-indentation to access to the local mechanical behaviour of the constitutive material of the stackings. Complementary analyses are in progress, through the modelling of micro-indentation experiments using the finite-element method, to improve the estimate of both elastic and plastic properties of the constitutive material thanks to an inverse approach [9].

\subsection{Characterization of the mechanical properties of the braze}

Micro-indentation has been used to characterize the mechanical behaviour of the eutectic structure constituting the braze joints. Indeed, if the eutectic structure has a specific mechanical behaviour, it could be useful to improve the modelling of cellular structures to know it. Indentation measurements have been performed on tube stackings, which have been elaborated following both treatments STT and LTT, in the constitutive tubes but also in the joints. As previously, indentations have been performed in a cross-section of the stacking, i.e., coaxially to the revolution axes of the tubes.

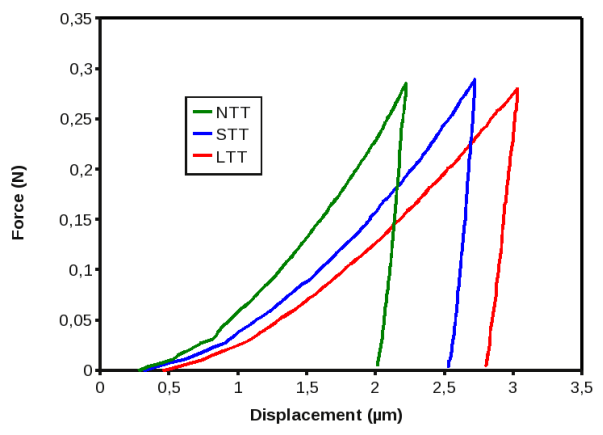

(a)

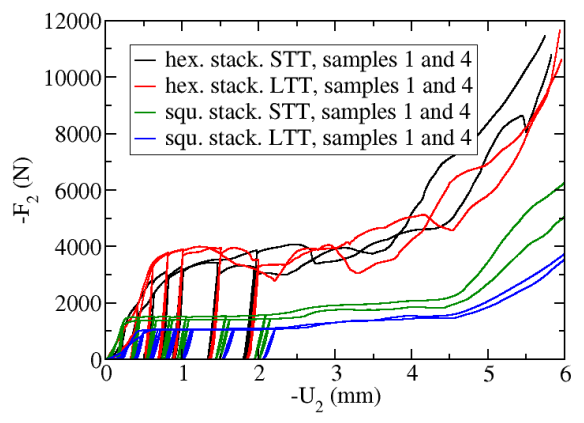

(b)

Fig. 4. Influence of the thermal treatment on a) the indentation curves b) the strength of the tube stackings.

Whereas results obtained on the isolated tubes were encouraging, hardness values and particularly Young's moduli measured in the walls of the tubes in the stackings were significantly higher than the ones obtained on the isolated tubes. Hardness values measured in the stackings equal 1.4 and 1.9 GPa for treatments STT and LTT, respectively, which can be considered rather consistent with the values measured on isolated tubes (see tab. 1). Corresponding Young's moduli estimated according to the method described before equal 226.0 and $275.0 \mathrm{GPa}$, respectively. Whatever the thermal treatment, these values are considerably higher than the ones estimated from the micro-indentation measurements on the isolated tubes. Such a disagreement could be explained by the diffusion of the phosphorus of the brazing deposit in the walls of the tubes. According to [12], diffusion of P results in stiffening phosphides. To validate this assumption, complementary analyses are in progress on isolated NiP coated tubes in which phosphorus has diffused, involving both mechanical and microstructural characterizations.

Nevertheless, as regards the behaviour of the braze, micro-hardness measurements have been carried out in the joints too on the stackings. Hardness values measured in the joints equal 4.5 and 3.0 $\mathrm{GPa}$, respectively, and corresponding estimated Young's moduli equal 276.0 and 289.0 GPa. These values are significantly higher than the ones obtained in the walls of the tubes, this is particularly true 
for the hardness. If indentation curves are studied in detail, two distinct behaviours are put in evidence in the joints, whatever the thermal treatment. The intermetallic compound $\mathrm{Ni}_{3} \mathrm{P}$ is the harder. Thanks to micro-indentation, the hardness of each phase has been measured. Whereas it equals $2.1 \mathrm{GPa}$ in the phase rich in $\mathrm{Ni}$, it equals $6.9 \mathrm{GPa}$ in the intermetallic compound for the treatment STT (1.9 and 5.7 GPa for the treatment LTT, respectively). Indeed the track is smaller in the intermetallic compound than in the other phases (the one rich in $\mathrm{Ni}$ and the Inconel ${ }^{\circledR}$ 600). Conversely the Young's modulus of $\mathrm{Ni}_{3} \mathrm{P}$ is systematically lower. Young's moduli equal 303.0 and $254.0 \mathrm{GPa}$ for the treatment STT, and 302.0 and $261.0 \mathrm{GPa}$ for the treatment LTT, for the phase rich in Ni and the intermetallic compound, respectively.

\section{Overall mechanical behaviour of the tube stackings}

To illustrate the final aim of these work, i.e., the improvement of the prediction of the overall mechanical properties of cellular structures thanks to a more accurate knowledge of the mechanical properties of their constitutive materials, the small sandwiches described in sec. 2 have been tested under uniaxial compression. Compressive loadings were applied on the top of the sandwiches, and were governed in displacement at a rate of $-0.5 \mathrm{~mm} / \mathrm{min}$. Force $v s$. displacement curves are plotted in figure 4(b). They show the good reproducibility of the tests. It is worth noting the hexagonal stacking is stiffer than the square one. Whereas no significant effect of the thermal treatment on the strength of the sandwich is observed in the case of the hexagonal stacking, for the square stacking the level of the densification plateau substantially falls for the longer thermal treatment.

These compression tests have been modelled in 2D, using the finite-element software ZSet [13, 14]. Thanks to the symmetries, only one quarter of the sandwiches was simulated each time applying planar boundary conditions on the symmetrical faces. Stackings were meshed using three quadratic tetrahedrons in the tube thickness and meshes were refined around the joint ends. The achievement of the convergence of the numerical problem has been checked. The contact between the neighbour tubes occurring during the compaction of the stacking has been modelled through a Coulomb contact without any penalty condition (fig. 5(a)). Similar numerical parameters and assumptions have been chosen for the FEM calculations previously described (sec. 3.1).

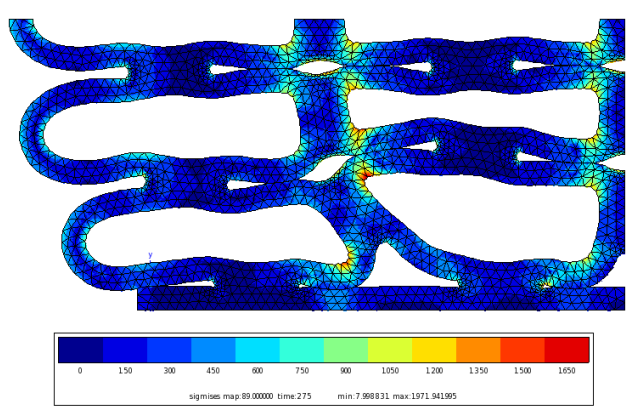

(a)

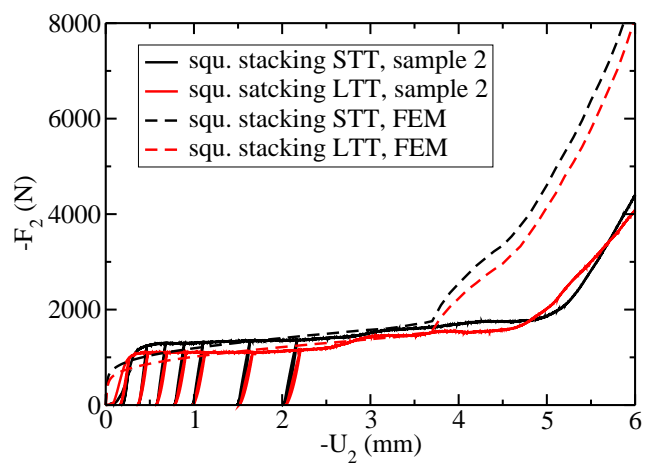

(b)

Fig. 5. a) von Mises stress map, for the sandwich with a square stacking core and the thermal treatment STT, for a global strain level around $60 \%$ b) comparison between the experimental compression curves and the predicted ones for the sandwiches with a square stacking core.

For the sake of simplicity, here the constitutive material of the sandwiches has been assumed to be homogeneous in the tubes, the joints and the sheets. As a first approximation the mechanical properties of the constitutive material were the ones identified from the tensile tests on the isolated tubes, see tab. 1. Calculations have been conducted up to a global strain level of $60 \%$. Figure 5(b) illustrates the 
comparison between the experimental and the predicted force $v s$. displacement curves for the square stacking, for treatments STT and LTT. These first results are promising since they show a good agreement between the experiment and the modelling. Especially, the level of the densification plateau is correctly predicted by the finite-element modelling. The elastic domain and the beginning of the plastic one are underestimated by the modelling but, according to the micro-hardness measurements performed on the sandwich structures, the mechanical properties of the in-situ constitutive material, especially the Young's moduli, seem to be higher than the ones determined on the isolated tubes because of the diffusion of the phosphorus. Moreover, the non-linearity experimentally observed at the start of the compaction because of parallelism defects of the sheets, hence the contact with the compression plates occurs progressively, makes difficult the measurement of the effective Young's modulus. Predicted Young's moduli are scheduled to be compared with the slopes of the elastic unloadings.

\section{Conclusions}

This work highlighted the significant influence of the process route on the mechanical properties of cellular structures. Especially, when high temperatures and added components are required for the elaboration or the assembly of the constitutive cells, the mechanical behaviour of the materials of which they are constituted can be strongly altered. In order to access to the local in-situ mechanical properties of the constitutive materials, micro-indentation experiments seem to be a very useful and relevant method. Even if the issue of the phosphorus diffusion in the stackings has been raised, as concerns the isolated tubes the moduli estimated from the micro-hardness measurements show a good agreement with the ones identified from the macroscopic tensile tests. Micro-hardness measurements performed in the joints also highlighted how sensitive is the method. Thanks to these experimental data, the overall mechanical behaviour of small sandwich structures has been predicted with a rather good accuracy.

To achieve the final goal of this work, the comparison between experiment and modelling will be improved step by step as soon as the influence of the phosphorus would have been tackled with, and then when reliable mechanical properties would have been identified for the braze material.

\section{References}

1. A. G. Evans, J. W. Hutchinson, M. F. Ashby, Multifunctionality of cellular metal systems (Harvard University, Cambridge, 1997).

2. W. S. Sanders, L. J. Gibson, Materials Science and Engineering, A347, (2003) 70-85.

3. W. S. Sanders, L. J. Gibson, Materials Science and Engineering A352, (2003) 150-161.

4. T. Fiedler, A. Öchsner, J. Grácio, Materials Science Forum 539-543, (2007) 1874-1879.

5. O. Friedl, C. Motz, H. Peterlik, S. Puchegger, N. Reger, R. Pippan, Metallurgical and Materials Transactions B 39B, (2008) 135-146.

6. O. Caty, E. Maire, S. Youssef, R. Bouchet, Acta Materialia 56, (2008) 5524-5534.

7. V. Marcadon, F. Feyel, Computational Materials Science 47, (2009) 599-610.

8. W. C. Oliver, G. M. Pharr, Journal of Materials Research 7(6), (1992) 1564-1583.

9. A. Villemiane, Analyse du comportement mécanique d'alliages pour couches de liaison de barrière thermique par microindentation instrumentée à haute température (PhD Thesis, in French, INP Lorraine, 2008).

10. D. Tabor, Philosophical Magazine A 74(5), (1996) 1207-1212.

11. K. L. Johnson, Journal of Machanics and Physics of Solids 18, (1970) 115-126.

12. R. K. Saha, T. I. Khan, AZo Journal of Materials Online 2, (2006) DOI: 10.2240/azojomo0225.

13. ZSet user manuel, version 8.3.6 (2006).

14. ZSet guide for material behaviour laws, version 8.3.6 (2006). 